\title{
Influence of percutaneous pulmonary valve implantation on exercise capacity: Which group of patients benefits most from the intervention?
}

\author{
Elżbieta Katarzyna Biernacka ${ }^{1}, E_{\text {wa Piotrowicz }}^{2}$, Aneta Fronczak ${ }^{3}$, Magdalena Mazgaj ${ }^{3}$, \\ Marcin Demkow ${ }^{4}$, Witold Rużyło ${ }^{4}$, Mateusz Śpiewak ${ }^{5}$, Mirosław Kowalski ${ }^{1}$, \\ Ryszard Piotrowicz ${ }^{6}$, Krzysztof Weroński ${ }^{1}$, Piotr Hoffman ${ }^{1}$ \\ ${ }^{1}$ Department of Congenital Heart Diseases, Institute of Cardiology, Warsaw, Poland \\ ${ }^{2}$ Telecardiology Center, Institute of Cardiology, Warsaw, Poland \\ ${ }^{3}$ Students' Research Group 'Heart Rhythm', Institute of Cardiology, Warsaw, Poland \\ ${ }^{4}$ Department of Coronary Artery Disease and Structural Heart Diseases, \\ Institute of Cardiology, Warsaw, Poland \\ ${ }^{5}$ Cardiac Magnetic Resonance Unit, Institute of Cardiology, Warsaw, Poland \\ ${ }^{6}$ Department of Cardiac Rehabilitation and Noninvasive Electrocardiology, \\ Institute of Cardiology, Warsaw, Poland
}

\begin{abstract}
Background: The aim of the study was to evaluate the role of cardiopulmonary exercise testing (CPET) parameters in assessing exercise capacity improvement after percutaneous pulmonary valve implantation (PPVI). Additionally, it aimed to determine if there are any baseline characteristics influencing that change.

Methods and results: The study comprised 32 patients (mean age $26 \pm 9$ ); $53 \%$ males; diagnosis: tetralogy of Fallot $(n=18)$, pulmonary atresia $(n=6)$, Ross procedure $(n=4)$, other (transposition of great arteries, pulmonary stenosis, double outlet right ventricle, common arterial trunk type II $-n=4$ ) who underwent successful PPVI due to right ventricular outflow tract dysfunction (predominant pulmonary regurgitation $-n=17$, predominant pulmonary stenosis $-n=15)$. Treadmill CPET was performed before and a year after PPVI along with clinical evaluation, cardiac magnetic resonance and transthoracic echocardiography. Twelve months post successful PPVI (pulmonary valve competence restoration and pulmonary gradient reduction from $58.8 \pm 47.1$ to $26.6 \pm 10.8 \mathrm{~mm} \mathrm{Hg}$ ) there was a significant decrease in the ventilatory equivalent for $\mathrm{CO}_{2}$ at peak exercise $\left(E Q C \mathrm{O}_{2}\right)(25.3 \pm 3.3$ to $24.3 \pm 3.0$, $p=0.04)$ and oxygen consumption at peak exercise $\left(p V \mathrm{O}_{2}\right)(20.4 \pm 5.0$ to $22.6 \pm 5.3 \mathrm{~mL} /$ $/ \mathrm{kg} / \mathrm{min}, p=0.04)$. Improved $E Q \mathrm{CO}_{2}$ correlated with an increase in right and left ventricular ejection fraction (respectively $R=-0.57, p=0.002 ; R=-0.56, p=0.002$ ). In this study, no baseline factors that might affect improvement in exercise function were found.

Conclusions: Successful PPVI leads to an improvement in exercise capacity and hemodynamic response to exercise. The correlation between the improvement in $\mathrm{EQCO}_{2}$ or peak $\mathrm{VO}_{2}$ and baseline characteristics was too weak to reliably identify the group of patients that will benefit from the procedure. (Cardiol J 2015; 22, 3: 343-350)
\end{abstract}

Key words: percutaneous pulmonary valve implantation, cardiopulmonary exercise testing, grown up congenital heart disease

Address for correspondence: Elżbieta Katarzyna Biernacka, MD, PhD, Professor, Department of Congenital Heart Diseases, Institute of Cardiology, ul. Alpejska 42, 04-628 Warszawa, Poland, tel: +48 223434 634, fax: +48 223434546 ,

e-mail:k.biernacka@ikard.pl

Received: 11.07.2014 Accepted: 27.01.2015 


\section{Introduction}

Patients with certain types of congenital heart defect need to undergo surgical repair of right ventricular (RV) outflow tract (RVOT) in their early infancy. After some years, the implanted RVOT conduits almost inevitably degenerate causing $\mathrm{RV}$ obstruction and/or regurgitation, which leads to progressive dysfunction of the $\mathrm{RV}$ requiring further surgical interventions. Consequently, those patients need to be subjected to multiple surgeries, each connected with an increasing risk. Percutaneous pulmonary valve implantation (PPVI) is a promising alternative for this group of patients. Some improvement of hemodynamic parameters after the procedure has been reported in several trials [1-4]. However, it is difficult to determine optimal timing for the intervention. Impaired physical capacity is reported to be the one of the first signs of decline in the RV function in asymptomatic patients [5, 6]. The aim of this study was to investigate whether PPVI leads to an improvement in exercise function and which patients might benefit from the procedure when assessed with selected cardiopulmonary exercise testing (CPET) parameters.

\section{Methods}

\section{Patient selection}

It is a retrospective cohort study. Out of 37 consecutive patients who underwent successful PPVI in the Institute of Cardiology in Warsaw, Poland, in years 2008-2012, 32 patients were investigated. The qualification of patients included: clinical examination, transthoracic echocardiography (TTE), cardiovascular magnetic resonance (CMR) and CPET performed before and 1 year after PPVI. Five patients were excluded due to inappropriate CPET performance (different protocol for a particular subject has been used before and after PPVI).

The study group consisted of 17 men and 15 women who underwent surgical repair of congenital heart defect in the past and were qualified for PPVI due to pulmonary valve dysfunction. The group was divided into two subgroups basing on the type of dominant lesion. Group 1 consisted of patients with predominant pulmonary stenosis (PS), significant pulmonary gradient, the pulmonary regurgitation fraction $(\mathrm{PRF})<25 \%$, and maximally mild PR in echocardiography. Group 2 consisted of patients with predominant pulmonary regurgitation (PR), PRF $\geq 25 \%$ or significant PR in echocardiography. Inclusion criteria for PPVI were based on surgical indications for RVOT reoperation [7]. Percutaneous pulmonary valve implantation using either Melody ${ }^{\circledast}$ valve $(n=20)$ or SAPIEN ${ }^{\mathrm{TM}}$ THV $(\mathrm{n}=12)$ was performed according to the protocol described previously $[1,8]$.

\section{Study protocol}

The study was approved by the local Research Ethics Committee. CPET, CMR and TTE were performed 1 day -5 months before PPVI and 1 year after the procedure along with the assessment of the New York Heart Association (NYHA) functional class. Each subject gave a signed informed consent.

\section{Cardiopulmonary exercise testing}

Cardiopulmonary exercise treadmill test was performed approximately $2 \mathrm{~h}$ after medications and a light breakfast in all patients. Each subject performed a symptom limited CPET according to a Ramp protocol or modified Bruce protocol (always the same protocol for a particular subject before and after PPVI so that each patient would be a control for him/herself). The test was performed using a Schiller treadmill (Carrollton, USA) which was connected to a computerized breath-by-breath spiroergometry system (ZAN 600, ZAN Messgeräte $\mathrm{GmbH}$, Germany).

Oxygen consumption $\left(\mathrm{VO}_{2}\right)$ was measured continuously using breath-by-breath analysis and used as an index of exercise capacity. Peak $\mathrm{VO}_{2}$ was defined as the highest oxygen uptake level achieved during the final $30 \mathrm{~s}$ of CPET. The formula used for the prediction of $\mathrm{VO}_{2}$ (in $\mathrm{mL} / \mathrm{kg} / \mathrm{min}$ ) was the Wasserman standard calculation, which incorporates sex, age, height, and weight of the subject and is valid for patients aged over 20 years [9]. Twelve-lead electrocardiogram (ECG) and heart rate (HR) were recorded continuously at rest, during CPET and during recovery until HR, ECG, and $\mathrm{VO}_{2}$ returned to the baseline values. Blood pressure (BP) was measured manually every $2 \mathrm{~min}$ using a sphygmomanometer.

Subjects were encouraged to exercise until they reached a self-determined limit of their functional capacity (perceived exertion or dyspnea) or until a physician terminated the test according to the European Society of Cardiology guidelines [10].

All CPET procedures were supervised by a nurse and a cardiologist. The parameters measured were the duration of workload, peak $\mathrm{VO}_{2}[\mathrm{~mL} / \mathrm{kg} /$ $/ \mathrm{min}], \%$ predicted peak $\mathrm{VO}_{2}$, minute ventilation (VE [L/min]), respiratory exchange ratio, $\mathrm{HR}$ [bpm], BP $[\mathrm{mm} \mathrm{Hg}]$, breathing frequency [L/min], and ventilatory equivalents for $\mathrm{CO}_{2}\left(\mathrm{EQCO}_{2}\right)$. 
Table 1. Demographic data.

\begin{tabular}{lcc}
\hline Parameter & & Total population (n=32) \\
\hline Age [years] & & $26 \pm 9$ \\
Body mass index $\left[\mathrm{kg} / \mathrm{m}^{2}\right]$ & & 21.5 \\
Sex (male/female) & II & $17 / 15$ \\
Baseline NYHA: & III & 8 \\
& IV & 15 \\
& Tetralogy of Fallot & 9 \\
Baseline diagnosis: & Pulmonary atresia & 0 \\
& Aortic valve stenosis, Ross procedure & 6 \\
& TGA, Rastelli procedure & 4 \\
& Pulmonary stenosis & 1 \\
& Double outlet right ventricle & 1 \\
& Common arterial trunk type I & 1 \\
Valve implanted: & Melody ${ }^{\circledR}$ valve & 1 \\
& SAPIEN ${ }^{\text {M }}$ THV & 20 \\
Predominant lesion: & Stenosis & 12 \\
& Regurgitation & 15 \\
\hline
\end{tabular}

NYHA — New York Heart Association functional classification; TGA - transposition of great arteries

\section{Cardiovascular magnetic resonance}

The data derived from CMR were collected using a $1.5 \mathrm{~T}$ scanner (Avanto, Siemens, Erlangen, Germany). The acquisition included steady-state free precision images in left ventricle (LV) long axis (2-, 3- and 4-chamber views) as well as a stack of short axis cross sectional views covering the ventricles from base to apex and phase contrast flow according to the instructions described previously [11]. After manual determination of end-diastolic and end-systolic endocardial outline, dedicated software (MASS 6.2.1, Medis, Leiden, the Netherlands) was used to assess RV and LV volumes and sizes. All volumes were indexed by body surface area (BSA) and expressed in $\mathrm{mL} / \mathrm{m}^{2}$. The degree of PRF was calculated with the dedicated software (Argus, Siemens, Erlangen, Germany).

\section{Transthoracic echocardiography}

Transthoracic echocardiography was performed using an echocardiographic system (Vivid 7, GE Medical Systems, Milwaukee, USA). The degree of RVOT gradient was derived from the peak velocity obtained by continuous-wave Doppler using the modified Bernoulli equation. Pulmonary regurgitation was evaluated on the basis of vena contracta. The regurgitant jet exceeding $6 \mathrm{~m}$ in width was found to be significant. The remainder was considered to be small or moderate.

\section{Statistical analysis}

All continuous variables are expressed as mean \pm standard deviation. Paired Student's t-test was used to compare changes in CPET, CMR, TTE parameters for normally distributed variables before and after PPVI. Non-normally distributed variables were compared with the aid of the Signed Rank test. The normality of distribution was assessed using the Shapiro-Wilk test. Correlations between changes in CPET parameters and (a) baseline CMR and TTE parameters, (b) changes in CMR and TTE were tested using Pearson's test. The level of significance was defined at $p<0.05$. All statistical analyses were performed with the use of MedCalc 10.0.2.0 statistical software (MedCalc, Mariakerke, Belgium).

\section{Results}

The patients' characteristics are shown in Table 1. Thirty-two patients met the inclusion criteria for the study. The mean age at the study onset was $26 \pm 9$ years; 17 patients were diagnosed with predominant regurgitation, 15 with predominant stenosis. The most common primary diagnosis was tetralogy of Fallot (56\%) followed by pulmonary atresia $(n=6)$, aortic valve disease (Ross procedure) $(\mathrm{n}=4)$, transposition of great arteries $(n=1)$, pulmonary stenosis $(n=1)$, double outlet RV $(n=1)$, common arterial trunk 


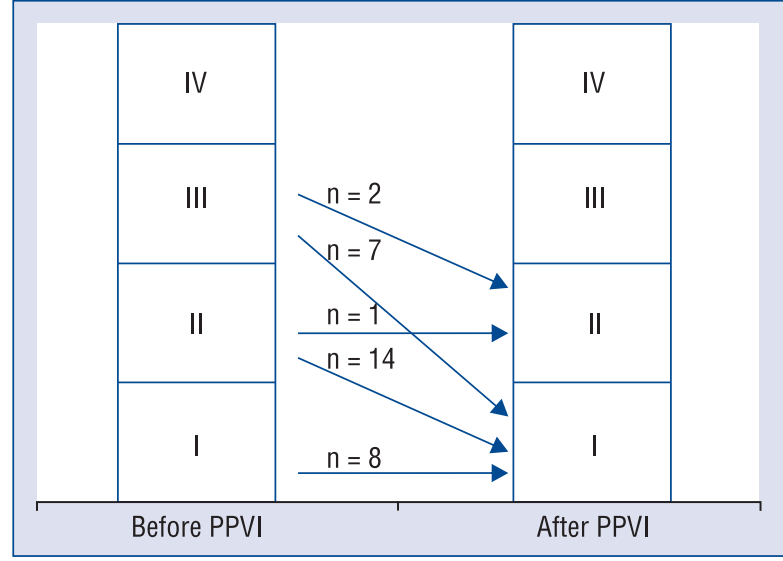

Figure 1. New York Heart Association (NYHA) functional class before and after percutaneous pulmonary valve replacement (PPVI); $\mathrm{n}$ - number of patients.

type I $(n=1)$. The patients had an average of two surgeries prior to PPVI. The mean time since the last intervention was 14.7 years. PPVI led to valve competence restoration observed in all patients 1 day after the procedure.

In the subgroup of patients with significant PS, pulmonary gradient decreased from 94.5 (range $41-200)$ to 38.9 (range 22-68). These results remained stable in a 1-year observation.

Additionally, we detected a statistically significant decrease in RV end-diastolic volume (RVEDV) and end-systolic volume (RVESV) and an increase in RV ejection fraction (EF) $(\mathrm{p}=0.003 ; \mathrm{p}=0.0003$; $\mathrm{p}=0.008$, respectively). When comparing the group of patients with PS with patients with PR, it appeared that the statistically significant decrease in RVEDV was noted in the latter group only. The end-diastolic volume of LV and LVEF increased (respectively $\mathrm{p}<0.0001 ; \mathrm{p}=0.04$ ).

Twelve months after PPVI no worsening in NYHA functional class was observed in any patient.

Furthermore, every symptomatic patient (with NYHA functional class II or above) who underwent the procedure improved in his/her functional class. The remaining 8 asymptomatic patients claimed significant improvement in physical capacity (Fig. 1).

PPVI led to a statistically significant increase in peak $\mathrm{VO}_{2}(20.4 \pm 5.0$ vs. $22.6 \pm 5.3 \mathrm{~mL} / \mathrm{kg} /$ $/ \mathrm{min} ; \mathrm{p}=0.04)$ (Fig. $2 \mathrm{~A}$ ). There was also a reduction in $\mathrm{EQCO}_{2}$ at peak oxygen uptake $(25.3 \pm 3.3$ vs. $24.3 \pm 3.0 ; \mathrm{p}=0.04)$. After comparing PR and PS groups, a statistically significant reduction in $\mathrm{EQCO}_{2}$ was observed only in PS group (25.1 \pm \pm 2.5 vs. $23.4 \pm 2.4 ; \mathrm{p}=0.03$ ) (Fig. $2 \mathrm{~B}$ ). Systolic $\mathrm{BP}$ at maximal effort increased after the procedure $(135.8 \pm 26.3$ vs. $154.1 \pm 29.5 \mathrm{~mm} \mathrm{Hg}$; $\mathrm{p}<$ $<0.0001)$. The duration of workload was elongated after the intervention ( $621 \pm 204$ vs. $676 \pm 182 \mathrm{~min}$; $\mathrm{p}=0.0004$ ).

There was no correlation between improvement in peak $\mathrm{VO}_{2}$ and either of the examined baseline CMR or TTE characteristics (Table 2).

An improvement in $\mathrm{EQCO}_{2}$ (lower values of $\triangle \mathrm{EQCO}_{2}$ ) correlated with baseline: greater mass of RV or LV, higher RVOT maximal gradient and PRF $(\mathrm{R}=-0.38 ; \mathrm{p}=0.047 ; \mathrm{R}=-0.35 ; \mathrm{p}=0.06$; $\mathrm{R}=-0.46 ; \mathrm{p}=0.01 ; \mathrm{R}=-0.35 ; \mathrm{p}=0.08$, respectively) (Table 2). The primary outcome defined as an improvement in $\mathrm{EQCO}_{2}$ (decrease in $\mathrm{EQCO}_{2}$

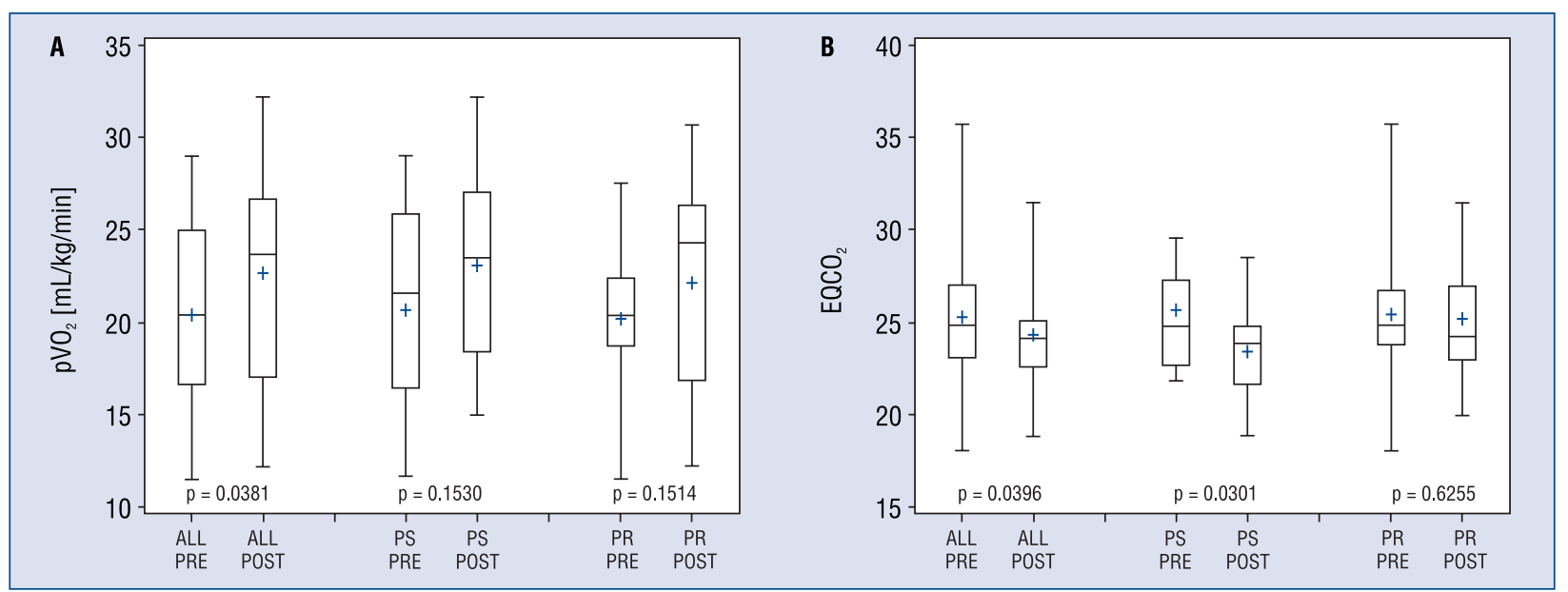

Figure 2. Results of peak oxygen uptake $\left(\mathrm{pVO}_{2}\right)$ and ventilatory equivalent for oxygen consumption $\left(\mathrm{EQCO}_{2}\right)$ before and 12 months after percutaneous pulmonary valve replacement (PPVI); PS — predominant stenosis; PR — predominant regurgitation. 
Table 2. Relationship between change in peak oxygen consumption $\left(\mathrm{VO}_{2}\right)$ and ventilatory equivalents for $\mathrm{CO}_{2}\left(\mathrm{EOCO}_{2}\right)$ and baseline magnetic resonance imaging and echocardiography parameters.

\begin{tabular}{|c|c|c|c|c|c|c|}
\hline & \multicolumn{3}{|c|}{$\Delta \mathrm{pVO}_{2}$} & \multicolumn{3}{|c|}{$\Delta \mathrm{EQCO}_{2}$} \\
\hline & $\mathbf{N}$ & $\mathbf{R}$ & $\mathbf{P}$ & $\mathbf{N}$ & $\mathbf{R}$ & $\mathbf{P}$ \\
\hline Right ventricular end-diastolic volume index $\left[\mathrm{mL} / \mathrm{m}^{2}\right]$ & 28 & 0.25 & NS & 28 & 0.07 & NS \\
\hline Right ventricular end-systolic volume index $\left[\mathrm{mL} / \mathrm{m}^{2}\right]$ & 28 & 0.17 & NS & 28 & 0.04 & NS \\
\hline Right ventricular ejection fraction [\%] & 28 & -0.07 & NS & 28 & 0.19 & NS \\
\hline Right ventricular mass $\left[\mathrm{ml} / \mathrm{m}^{2}\right]$ & 28 & 0.13 & NS & 28 & -0.38 & 0.047 \\
\hline Left ventricular end-diastolic volume index $\left[\mathrm{mL} / \mathrm{m}^{2}\right]$ & 28 & -0.23 & NS & 28 & -0.17 & NS \\
\hline Left ventricular end-systolic volume index $\left[\mathrm{mL} / \mathrm{m}^{2}\right]$ & 28 & -0.08 & NS & 28 & -0.18 & NS \\
\hline Left ventricular ejection fraction [\%] & 28 & -0.13 & NS & 28 & 0.14 & NS \\
\hline Left ventricular mass $\left[\mathrm{mL} / \mathrm{m}^{2}\right]$ & 28 & -0.24 & NS & 28 & -0.35 & 0.06 \\
\hline Right ventricular outflow tract maximal gradient & 28 & -0.04 & NS & 28 & -0.46 & 0.01 \\
\hline Pulmonary regurgitation fraction [\%] & 26 & -0.03 & NS & 26 & -0.35 & 0.08 \\
\hline
\end{tabular}

$\mathrm{N}$ - number of patients; $\mathrm{R}$ - Pearson correlation coefficient; $\Delta$ - indicates change (from baseline to 1 year post implantation); NS — statistically non-significant change or no trend; Bold indicates statistically significant change or trend

Table 3. Univariate regression analysis of potential predictors of changes inpeak oxygen consumption $\left(\mathrm{VO}_{2}\right)$ uptake and ventilatory equivalents for $\mathrm{CO}_{2}\left(\mathrm{EOCO}_{2}\right)$.

\begin{tabular}{|c|c|c|c|c|c|c|}
\hline & \multicolumn{3}{|c|}{$\triangle \mathrm{pVO}_{2}$} & \multicolumn{3}{|c|}{$\triangle \mathrm{EOCO}_{2}$} \\
\hline & $\mathbf{N}$ & $\mathbf{R}$ & $\mathbf{P}$ & $\mathbf{N}$ & $\mathbf{R}$ & $\mathbf{P}$ \\
\hline$\Delta$ Right ventricular end-diastolic volume index $\left[\mathrm{mL} / \mathrm{m}^{2}\right]$ & 28 & -0.35 & 0.07 & 28 & 0.12 & NS \\
\hline$\Delta$ Right ventricular end-systolic volume index $\left[\mathrm{mL} / \mathrm{m}^{2}\right]$ & 28 & -0.25 & NS & 28 & 0.20 & NS \\
\hline$\Delta$ Right ventricular ejection fraction [\%] & 28 & 0.02 & NS & 28 & -0.57 & 0.002 \\
\hline$\Delta$ Right ventricular mass $\left[\mathrm{mL} / \mathrm{m}^{2}\right]$ & 26 & -0.15 & NS & 26 & 0.27 & NS \\
\hline$\Delta$ Left ventricular end-diastolic volume index $\left[\mathrm{mL} / \mathrm{m}^{2}\right]$ & 28 & 0.09 & NS & 28 & -0.01 & NS \\
\hline$\Delta$ Left ventricular end-systolic volume index $\left[\mathrm{mL} / \mathrm{m}^{2}\right]$ & 28 & 0.06 & NS & 28 & 0.43 & 0.02 \\
\hline$\Delta$ Left ventricular ejection fraction [\%] & 28 & -0.01 & NS & 28 & -0.56 & 0.002 \\
\hline$\Delta$ Left ventricular mass $\left[\mathrm{mL} / \mathrm{m}^{2}\right]$ & 27 & 0.40 & 0.04 & 27 & 0.36 & NS \\
\hline$\Delta$ Right ventricular outflow tract maximal gradient & 27 & 0.10 & NS & 27 & 0.44 & 0.02 \\
\hline
\end{tabular}

$\mathrm{N}$ - number of patients; R - Pearson correlation coefficient; $\Delta$ - indicates change (from baseline to 1 year post implantation); NS — statistically non-significant change or no trend; Bold indicates statistically significant change or trend

after the procedure) correlated significantly with an increase in EF of the RV and LV (Table 3).

\section{Discussion}

Optimal timing for the intervention in patients with RVOT dysfunction has been widely debated. The group of patients subjected to PPVI is varied in terms of primary diagnosis, symptoms, number of interventions, type of dominant lesion, and anatomy of the heart. Investigators try to define the baseline characteristics related to an improvement after PPVI, most typically acknowledged by the assessment of ventricular end-systole and end-diastole volumes, or CPET [11-14]. The reported factors that are believed to influence short- and/or long-term results include: type of dominant lesion
[4, 15]; baseline RVEDV [16, 17]; and baseline peak $\mathrm{VO}_{2}$ value [18]. In this study, no baseline factors that might affect an improvement in exercise function were found. This, however, might be due to small, heterogeneous patient group, therefore further research is needed.

Previous studies remain unequivocal about the influence of PPVI on exercise performance. Contrary to surgical replacement of pulmonary valve (PVR), PPVI does not involve any incisions in the myocardium - a frequent cause of a conduction block and arrhythmias, which worsen heart function [19]. PPVI leading to restoration of pulmonary valve function, similarly to PVR, simultaneously being a less invasive method, could be expected to have a positive effect on heart function and consequently exercise performance. There is, however, 


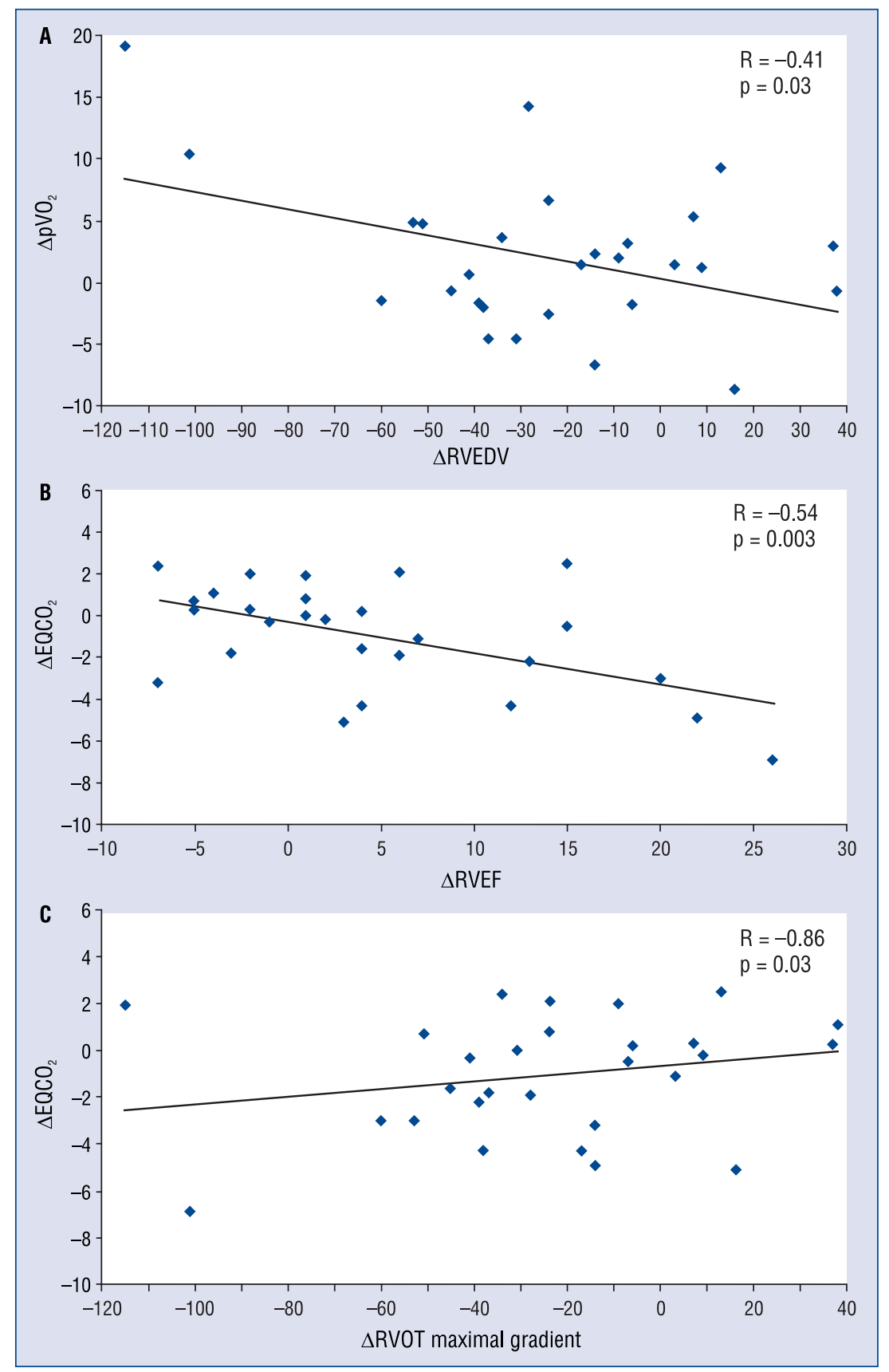

Figure 3. Results of peak oxygen uptake $\left(\mathrm{pVO}_{2}\right)$ and ventilatory equivalent for oxygen consumption $\left(\mathrm{EQCO}_{2}\right)$ before and 12 months after percutaneous pulmonary valve replacement (PPVI); $\Delta$ - indicates change (from baseline to 1 year post implantation); R - Pearson correlation coefficient; RVEDV — right ventricular end-diastolic volume; RVEF — right ventricular ejection fraction; RVOT — right ventricular outflow tract.

no clear evidence of improvement in cardiopulmonary function after the procedure [11, 15, 18, 20,21]. In our study, a small but significant improvement in peak $\mathrm{VO}_{2}$ and $\mathrm{EQCO}$ were reported.

In order to understand the outcomes of the conducted experiments, patients' magnetic resonance imaging and TTE were investigated and then the findings were correlated with CPET data. An important finding derived from the study was that the improvement in RV function following RVOT gradient reduction due to PPVI, led to exercise function improvement (Fig. 3). Healthy subjects respond to exercise by increasing $\mathrm{RV}$ pressure and pulmonary blood flow [22]. In patients with RVOT dysfunction there is poor exercise performance resulting from (1) submaximal pulmonary circula- 
tion, (2) pressure overload of RV. In line with this description and previous studies, we reported that in patients with congenital heart disease with pulmonary conduit dysfunction exercise capacity is significantly impaired $[18,23]$.

In terms of RV overload, patients with PR differ from patients with PS. PR leads to RV volume overload, observed as an increase in RVEDV that could be followed in our study. In patients with PS, however, abnormal loading conditions lead to an increase in RVESV with no change in RVEDV. In this group, PPVI leads to reduction in RVESV, with no change in RVEDV. The results are consistent with the findings by Coats et al. [11, 20].

Releasing RV from volume and pressure overload due to PPVI combined with increased RVEF might be assigned to a better pulmonary arterioles blood flow. Consequently, increased pre-load improves LVEF. The relocation of blood towards pulmonary circulation enhances the blood saturation, which combined with an increase in biventricular effective stroke volume, results in improved physical capacity (examined by change in peak $\mathrm{VO}_{2}$ and ventilatory equivalent for $\mathrm{CO}_{2}$ ). Also, the subjective component, reflected by the duration of workload in CPET and NYHA classification, clearly indicated an improvement in cardiopulmonary function. These findings are consistent with previous studies examining the correlation between changes in RV and LV functions and exercise capacity. Lurz et al. [4] described postprocedural improvement in peak oxygen uptake and minute ventilation/carbon dioxide elimination slope as a result of RV and LV effective stroke volume increase during exercise in patients with PS, which is consistent with our findings.

Our data suggest a clear positive influence of PPVI on peak $\mathrm{VO}_{2}$ and a link between peak $\mathrm{VO}_{2}$ and magnetic resonance imaging parameters connected with the right heart physiology. In our study, however, we observed a significant but small improvement in peak $\mathrm{VO}_{2}$. The cause of that might be a considerably small group of patients included in the study and different factors affecting peak $\mathrm{VO}_{2}$, such as: oxygen carrying capacity of the blood, peripheral blood flow, extraction by the tissue, pulmonary diseases, quantity of exercising muscles, fat-free mass. Another limitation of using peak $\mathrm{VO}_{2}$ in assessing exercise performance is the inaccuracy of measuring external work rate when performing a treadmill test. Peak $\mathrm{VO}_{2}$ is acknowledged as a gold standard for cardiorespiratory fitness. In clinical testing, however, we should make allowances for symptom limitation of exercise, which is often the case in patients with congenital heart disease.
An improvement in medical databases is typically defined with regard to standards. In the case of patients with congenital heart disease, however, the physiology of the heart differs from that in other individuals, moreover it changes during exercise. That is why acknowledging the indicators of "improvement" is not simple. In the case of patients with pulmonary regurgitation, we observed postprocedural reduction in RVEDV as a result of reduced blood retrograde flow through the pulmonary valve during diastole. That supports the findings by Coats et al. [11], who explained that RV in this group of patients is on decompensatory limb of the Starling curve at baseline which shifts leftwards back to the compensatory limb following PPVI. The group of patients with predominant stenosis show a different pattern of RV hemodynamics following the procedure. The decompensated state, contrary to what is seen in PR group, is a result of increased afterload. Intervention relieves abnormal loading conditions and consequently decreases RVESV. These findings are similar to the results reported by Coats et al. [20].

In order to describe "improvement" in all patients, independently of the type of dominant lesion, we chose to use CPET parameters, considering the fact that impaired physical capacity is reported to be the one of the first signs of decline in RV function in asymptomatic patients [5]. The improvement was assessed using: (1) peak $\mathrm{VO}_{2}$ - an important parameter defining the limits of the cardiopulmonary system [24]; (2) ventilatory response to exercise, defined as $\mathrm{EQCO}{ }_{2}\left(\mathrm{VE} / \mathrm{VCO}_{2}\right)$ at maximally obtained duration of exercise in both tests before and after PPVI. Both elements of the $\mathrm{EQCO}_{2}$ formula change with the duration of the exercise, which encouraged us to include the results at the same time point.

\section{Limitations of the study}

The main limitation of the study was a small group of patients. It is possible that increasing the study population might help find the factors associated with the post-procedural improvement. Additionally, the follow-up period was rather short so we were unable to comment on a long-outcome of the procedure (over 1 year). The mean respiratory exchange ratio was 0.98 , which indicated that in some patients, the physical effort was submaximal, consequently their results might have been underestimated.

The Wasserman standard formula, used for calculating predicted peak $\mathrm{VO}_{2}$ is valid for patients aged over 20 years. Five out of 29 patients included in the study were under the age of 20 years, which 
made us use peak $\mathrm{VO}_{2}$ instead of predicted peak $\mathrm{VO}_{2}$ for further calculations.

\section{Conclusions}

The results directed us to three major conclusions: (1) PPVI resulted in an improvement in the duration of workload, peak oxygen uptake and $\mathrm{EQCO}_{2}$. (2) Ventilatory equivalent for $\mathrm{CO}_{2}$ and peak $\mathrm{VO}_{2}$ were convenient for the assessment of post-procedural improvement. (3) The correlation between an improvement in $\mathrm{EQCO}_{2}$ or peak $\mathrm{VO}_{2}$ and baseline characteristics was too weak to reliably identify the group of patients that will benefit from the procedure.

Conflict of interest: Marcin Demkow - personal fees from Medtronic; other authors - no conflict of interests reported.

\section{References}

1. Demkow M, Biernacka EK, Spiewak M et al. Percutaneous pulmonary valve implantation preceded by routine prestenting with a bare metal stent. Catheterization Cardiovasc Intervent, 2011; 77: 381-389.

2. Eicken A, Ewert P, Hager A et al. Percutaneous pulmonary valve implantation: Two-centre experience with more than 100 patients. Eur Heart J, 2011; 32:1260-1265.

3. Kenny D, Hijazi ZM, Kar S et al. Percutaneous implantation of the Edwards SAPIEN transcatheter heart valve for conduit failure in the pulmonary position: Early phase 1 results from an international multicenter clinical trial. J Am Coll Cardiol, 2011; 58: 2248-2256.

4. Lurz P, Nordmeyer J, Giardini A et al. Early versus late functional outcome after successful percutaneous pulmonary valve implantation: are the acute effects of altered right ventricular loading all we can expect? J Am Coll Cardiol, 2011; 57: 724-731.

5. Friedberg MK, Fernandes FP, Roche SL et al. Relation of right ventricular mechanics to exercise tolerance in children after tetralogy of Fallot repair. Am Heart J, 2013; 165: 551-557.

6. Giardini A, Specchia S, Tacy TA et al. Usefulness of cardiopulmonary exercise to predict long-term prognosis in adults with repaired tetralogy of Fallot. Am J Cardiol, 2007; 99: 1462-1467.

7. Lurz P, Coats L, Khambadkone S et al. Percutaneous pulmonary valve implantation impact of evolving technology and learning curve on clinical outcome. Circulation, 2008; 117: 1964-1972.

8. Piotrowicz E, Biernacka EK, Mazgaj M et al. Electrocardiographic characteristics of the right ventricle following hemodynamic improvement after percutaneous pulmonary valve implantation, one year follow-up. J Electrocardiol, 2014; 47: 612-617.

9. Wasserman K. Principles of exercise testing and interpretation: Including pathophysiology and clinical applications. 4th Ed. Lippincott Williams \& Wilkins, Philadelphia 2005.

10. Piepoli MF, Corra U, Agostoni PG et al.; Task Force of the Italian Working Group on Cardiac R, Prevention, Working Group on Cardiac R, Exercise Physiology of the European Society of C.
Statement on cardiopulmonary exercise testing in chronic heart failure due to left ventricular dysfunction: Recommendations for performance and interpretation. Part II: How to perform cardiopulmonary exercise testing in chronic heart failure. Working Groups on Epidemiology \& Prevention and Cardiac Rehabilitation and Exercise Physiology. Eur J Cardiovasc Prevent Rehabilitation, 2006; 13: 300-311.

11. Coats L, Khambadkone S, Derrick G et al. Physiological consequences of percutaneous pulmonary valve implantation: The different behaviour of volume- and pressure-overloaded ventricles. Eur Heart J, 2007; 28: 1886-1893.

12. Vliegen HW, van Straten A, de Roos A et al. Magnetic resonance imaging to assess the hemodynamic effects of pulmonary valve replacement in adults late after repair of tetralogy of fallot. Circulation, 2002; 106: 1703-1707.

13. Therrien J, Siu SC, McLaughlin PR, Liu PP, Williams WG, Webb GD. Pulmonary valve replacement in adults late after repair of tetralogy of fallot: are we operating too late? J Am Coll Cardiol, 2000; 36: $1670-1675$

14. Therrien J, Provost Y, Merchant N, Williams W, Colman J, Webb G. Optimal timing for pulmonary valve replacement in adults after tetralogy of Fallot repair. Am J Cardiol, 2005; 95: 779-782.

15. Lurz P, Giardini A, Taylor AM et al. Effect of altering pathologic right ventricular loading conditions by percutaneous pulmonary valve implantation on exercise capacity. Am J Cardiol, 2010; 105: 721-726.

16. Oosterhof T, Vriend JW, Mulder BJ. Letter regarding article by Frigiola et al. "Pulmonary regurgitation is an important determinant of right ventricular contractile dysfunction in patients with surgically repaired tetralogy of Fallot". Circulation, 2005, 111: e112; author reply e112.

17. Frigiola A, Tsang V, Bull C et al. Biventricular response after pulmonary valve replacement for right ventricular outflow tract dysfunction: Is age a predictor of outcome? Circulation, 2008; 118 (14 suppl.): S182-S190.

18. Batra AS, McElhinney DB, Wang W et al. Cardiopulmonary exercise function among patients undergoing transcatheter pulmonary valve implantation in the US Melody valve investigational trial. Am Heart J, 2012; 163: 280-287.

19. Plymen CM, Bolger AP, Lurz P et al. Electrical remodeling following percutaneous pulmonary valve implantation. Am J Cardiol, 2011; 107: 309-314.

20. Coats L, Khambadkone S, Derrick G et al. Physiological and clinical consequences of relief of right ventricular outflow tract obstruction late after repair of congenital heart defects. Circulation, 2006; 113: 2037-2044.

21. Vezmar M, Chaturvedi R, Lee KJ et al. Percutaneous pulmonary valve implantation in the young 2-year follow-up. J Am Coll Cardiol Cardiovasc Intervent, 2010; 3: 439-448.

22. Hasan BS, Lunze FI, McElhinney DB et al. Exercise stress echocardiographic assessment of outflow tract and ventricular function in patients with an obstructed right ventricular-to-pulmonary artery conduit after repair of conotruncal heart defects. Am J Cardiol, 2012; 110: 1527-1533.

23. Zapletal A, Samanek M, Hruda J, Hucin B. Lung function in children and adolescents with tetralogy of Fallot after intracardiac repair. Pediatric Pulmonol, 1993; 16: 23-30.

24. Balady GJ, Arena R, Sietsema K et al. Clinician's Guide to cardiopulmonary exercise testing in adults: A scientific statement from the American Heart Association. Circulation, 2010; 122: 191-225. 